\title{
Off-line magnetic resonance imaging navigation of cervix cancer brachytherapy in patients with risk factors for uterine perforation
}

\author{
Assist. Prof. Noora Mohammed Al-Hammadi, MD, Suparna Halsnad Chandramouli, MD, Rabih Hammoud, \\ Primoz Petric, MD, PhD \\ Department of Radiation Oncology, National Center for Cancer Care and Research, Hamad Medical Corporation, Doha, Qatar
}

\begin{abstract}
Purpose: There are no reports on pre-insertion identification of cervix cancer patients at risk for uterine perforation during brachytherapy (BT). Our aim was to assess the incidence of risk factors in our patient cohort, and assess feasibility of a novel technique of magnetic resonance imaging (MRI)-guided navigation for applicator insertion (NAI) in high-risk cases.

Material and methods: All patients with locally advanced cervical cancer, treated with image guided adaptive BT at our department between October 2013 and June 2017 were considered for analysis. Tumor characteristics on initial MRI (MRI initial $\left._{1}\right)$, pre-BT MRI (MRI pre-BT ), and BT MRI (MRI $\left.\mathrm{BT}_{\mathrm{T}}\right)$ were assessed. Frequency of risk factors (age above 60 years, retroverted/retroflected uterus, tumor necrosis, non-visible cervical orifice, distorted cervical canal) was recorded. Patients with two or more factors underwent MRI guided NAI. Time needed for NAI was estimated and procedure feasibility score assigned using a three-tiered scoring system.

Results: Twenty-seven patients (98 insertions) were included. Mean tumor volume was $70.2( \pm 47.9), 17.8( \pm 18.9)$, and $10.3( \pm 9.1) \mathrm{cm}^{3}$ on $\mathrm{MRI}_{\text {initial, }}, \mathrm{MRI}_{\text {pre-BT}}$, and $\mathrm{MRI}_{\mathrm{BT} 1}$, respectively $(p<0.05)$. In $16(59 \%)$ cases, $\geq 1$ perforation risk factor was found on MRI $\mathrm{pre}_{\mathrm{BT}}$ : distorted canal in $12(44 \%)$, necrosis in $9(33 \%)$, retroverted/retroflected uterus in 8 (30\%) cases. Nine $(33 \%)$ patients had $\geq 2$ risk factors and underwent MRI guided NAI. Additional time to perform NAI was estimated at 105 minutes, and feasibility score was 1 in all cases. There were no cases of uterine perforation.

Conclusions: Using pre-insertion MRI, we found $\geq 2$ risk factors for uterine perforation in $1 / 3$ of patients. Off-line MRI navigation was feasible and enabled non-complicated insertion in all cases. Further studies with larger sample size are warranted to assess its clinical efficacy.

J Contemp Brachytherapy 2017; 9, 6: 519-526 DOI: https://doi.org/10.5114/jcb.2017.71912
\end{abstract}

Key words: brachytherapy, cervical cancer, cervix cancer, magnetic resonance imaging, uterine perforation.

\section{Purpose}

Image-guided adaptive brachytherapy (IGABT) is becoming increasingly adopted as a state of the art treatment component for locally advanced cervical cancer $[1,2$, $3,4,5,6,7]$. Accurate placement of applicators in the uterus, vagina, and parametria is required for favorable outcome of IGABT. During dilatation of cervical canal and insertion of the intrauterine tandem, radiation oncologist frequently encounters a challenging patho-anatomical situation. At time of BT, vaginal, cervical, and uterine architecture is commonly disfigured due to residual tumor, necrosis, therapy-induced tissue defects or fibrotic changes. In addition, bleeding or narrow vaginal anatomy can impede identification of external cervical orifice. These findings, especially when combined with posteriorly or laterally inclined and/or thin-walled uterus, increase the risk of false route and perforation during cervical dilatation and applicator insertion $[8,9]$.

Uterine perforation with the intracavitary applicator is reported to occur in up to $2-14 \%$ of insertions $[8,9,10,11]$. When unrecognized, it may result in over- and under-irradiation of pelvic organs and the tumor, respectively [10]. This leads to increased chance of radiotherapy side effects and reduced chance of cure [10]. Post-insertion sectional imaging enables identification of applicator false route and helps avoid these consequences [8]. However, prevention of uterine perforations is preferred over their early detection, because it also helps avoid other complications such as bleeding, infection, tumor seeding, delay of treatment, need for second anesthesia, etc. Real-time ultrasound has been recommended by several authors to guide 
the tandem insertion and reduce the risk of perforation $[8,11,12,13,14,15,16]$. At our department, ultrasound is not readily available in the BT suite. But in 2013, we implemented magnetic resonance imaging (MRI) based IGABT. In addition to MRI at diagnosis and at each BT fraction, pre-BT MRI (MRI ${ }_{\text {pre-BT }}$ ) is routinely obtained prior to applicator insertion in our practice. We use MRI $\mathrm{Mre}_{\mathrm{BT}}$ for pre-planning of combined intracavitary/interstitial (IC/ IS) implants and off-line navigation of applicator insertion (NAI). The aim of our study was to assess, in our patient cohort, the incidence of risk factors for uterine perforation, which were described by authors before $[8,9]$. We also aimed to describe and assess feasibility of our innovative pre-planning technique of off-line MRI-guided NAI.

\section{Material and methods}

Image data sets of all patients with pathology-proven cervical cancer, treated at our department with MRIbased IGABT between October 2013 and June 2017, were considered for analysis. Our routine imaging and treatment protocol was applied in all cases (Figure 1). Patients with available pre-treatment and pre-BT MRI, and complete medical records were included. This work was approved by the Institutional medical research Centre/ Ethical Committee (study number: MRC 17239/17).

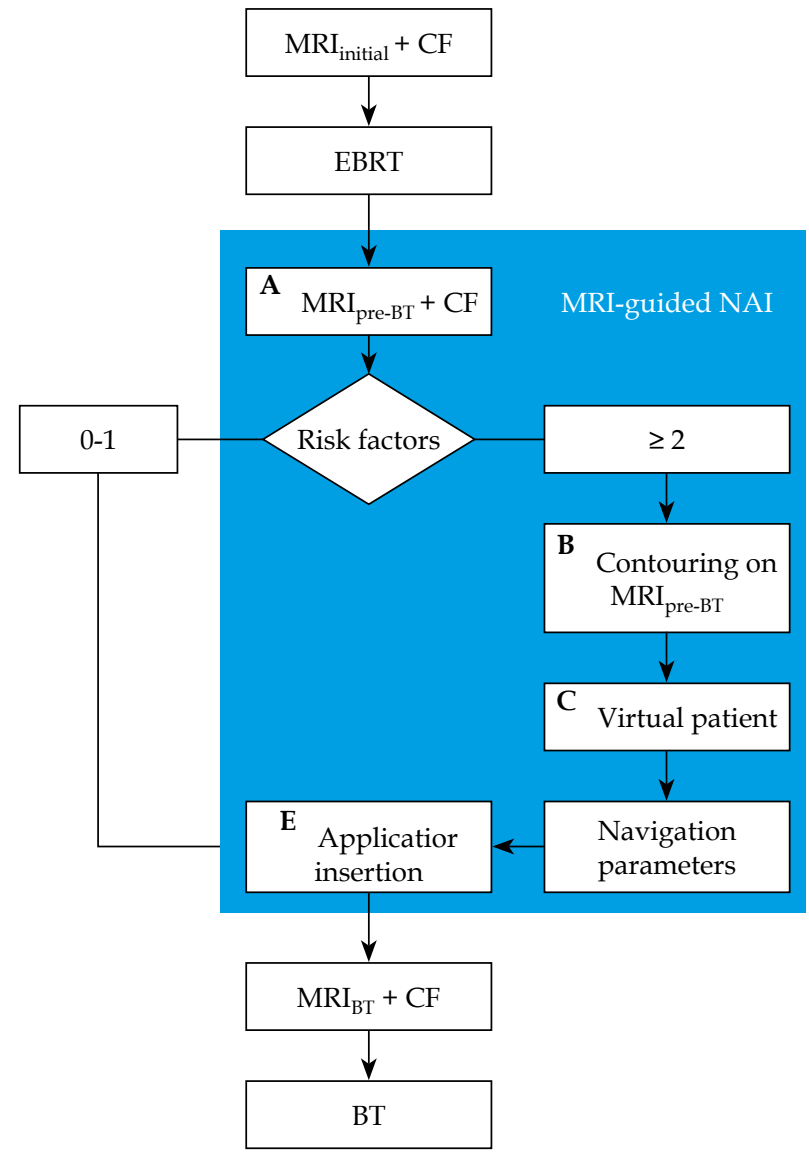

Fig. 1. Outline of our treatment protocol. Blue area (A-E): Magnetic resonance imaging (MRI) -guided navigation of applicator insertion (NAI). EBRT - external beam radiotherapy, BT - brachytherapy, CF - clinical findings

\section{External beam radiotherapy and brachytherapy}

External beam radiotherapy (EBRT) planning was based on computed tomography (CT), 18-fluorodeoxy glucose positron emission tomography (18-FDG PET), and MRI simulator. Volumetric modulated arc therapy or 3D conformal radiotherapy was used to apply 45-50.4 Gy in 25-28 daily fractions to the pelvis, with 6 or $15 \mathrm{MV}$ photon beams. In cases considered at high-risk for para-aortic nodal involvement, para-aortic region was treated to 45 Gy. For metastatic pelvic lymph nodes, we aimed to deliver 60-65 Gy, using simultaneous integrated and/or sequential boost $[17,18]$ with $2 \mathrm{~Gy}$ per fraction. Metastatic para-aortic lymph nodes were treated with individually adapted boost dose, depending on normal tissue dose constraints. Daily cone beam CT was used for image guidance. Concurrent cisplatin $\left(40 \mathrm{mg} / \mathrm{m}^{2}\right.$ per week for 5 weeks) was planned for all patients. Following completion of EBRT, patients were scheduled for 3 (before 2015) or 4 (since 2015) fractions of MRI based IGABT using IC \pm IS technique. In our practice, IGABT is performed twice weekly on outpatient basis, and is planned for the $6^{\text {th }}$ and $7^{\text {th }}$ week of treatment, aiming to keep the overall treatment time below 50 days. During BT dose optimization, linear quadratic model with $\alpha / \beta$ ratio of $10 \mathrm{~Gy}$ for tumor and $3 \mathrm{~Gy}$ for the organs at risk was applied to calculate the combined (EBRT and BT) biologically equivalent doses in 2 Gy per fraction $\left(\mathrm{EQD}_{2}\right)$. While respecting dose constraints for the normal tissues, our planning objective was to deliver a nominal D90 for the high-risk clinical target volume $\left(\mathrm{CTV}_{\mathrm{HR}}\right)$ [19] of $\geq 7$ Gy per IGABT fraction. This corresponds to a total EQD2 planning aim (EBRT + BT) of $\geq 85 \mathrm{~Gy}$.

\section{Magnetic resonance imaging technique}

Pelvic MRI was scheduled at different points in time (Figure 1): 1) $M R I_{\text {initial }}$ : prior to treatment start - at EBRT simulation; 2) MRI pre-BT $_{\text {in }} 4^{\text {th }}$ or $5^{\text {th }}$ week of EBRT; 3) $\mathrm{MRI}_{\mathrm{BT}}$ : at each fraction of BT with the applicator in place.

Imaging was performed on a dedicated wide-bore 1.5T 450w MRI simulator (General Electric Optima ${ }^{\circledR}$, Milwaukee, WI, USA) equipped with radiotherapy applications. Images were acquired using pelvic coils with patient in supine position [20]. For MRI $\mathrm{initial}_{\text {and }} \mathrm{MRI}_{\text {pre-BT, }}$ imaging was completed without BT applicator in place. For MRI $_{\text {pre-BT }}$, vaginal walls were unfolded by injecting aqueous gel into the vagina to improve visualization. For $\mathrm{MRI}_{\mathrm{BT}}$, the images were acquired after the BT procedure with MRI compatible applicator in place. Our protocol included T2 weighted FSE propeller, diffusion weighted ( $b$ values of 150 and 1000), non-contrast enhanced T1 and Dixon type LAVA-Flex sequences. Images were obtained with a slice thickness of $3 \mathrm{~mm}$ in para-transverse (perpendicular to cervical canal), para-sagittal, and para-coronal (parallel to cervical canal) orientation. Matrix size was $288 \times 288$ (para-axial and para-coronal), and $320 \times 256$ (para-sagittal). The field of view was covering the entire uterus, vagina, and tumor. For the T2 FSE sequence, system related geometric distortions after vendor-provid- 
ed correction algorithms are negligible for BT treatment planning, which was confirmed by our recent study [21]. Anonymized MRI data-sets were imported to the treatment planning system (TPS) Brachyvision (Varian, Medical Systems ${ }^{\circledR}$, Palo Alto, CA, USA) for interpretation and pre-planning.

\section{Interpretation of magnetic resonance imaging and clinical findings}

Magnetic resonance imaging was assessed by a senior radiation oncologist, experienced in image guided gynecological radiotherapy. Consultation with radiologist was available in case of equivocal findings. All clinical and imaging findings at diagnosis, before BT and at first BT fraction (BT1), were considered during MRI interpretation (Figure 1) [22]. Size and topography of the tumor, vagina, cervix, uterus, and residual pathological tissues were evaluated. The following details were recorded:

1. MRI $_{\text {initial}}, \mathrm{MRI}_{\text {pre-BT }}$ and MRI $\mathrm{BT1}_{1}$ : Gross tumor volume (GTV) was contoured using the TPS as the lesion demonstrating hyperintensity signal on T2w FSE sequence [19].

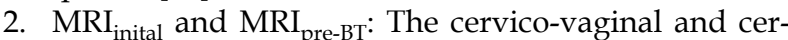
vico-uterine angles were measured (Figure 2), using the TPS tools.

3. $\mathrm{MRI}_{\text {pre-BT }}$ and pre-BT clinical examination: The frequency of findings of necrotic areas, non-visible cervical orifice, and distorted cervical canal were recorded (Figure 2). Distorted cervical canal was defined by two or more changes of canal direction or poorly-identifiable lumen.

4. Cone beam CT: When cervico-vaginal and/or cervico-uterine angle on $\mathrm{MRI}_{\text {pre-BT }}$ was $\geq 180^{\circ}$ (RVF-ret- roverted/retroflected uterus), the changes of uterine position during EBRT were analyzed by reviewing daily cone beam CT images.

\section{Magnetic resonance imaging-guided navigation of applicator insertion}

Information from clinical findings, $\mathrm{MRI}_{\text {initial, }}$ and $\mathrm{MRI}_{\text {pre-BT }}$ were considered during applicator insertion in all cases. Patients with $\geq 2$ risk factors for perforation according to [8] (age above 60 years, uterus in RVF, tumor necrosis, clinically non-visible cervical orifice, distorted cervical canal) underwent systematic MRI-guided NAI. Steps of the NAI pre-planning procedure are outlined in Figure 1. First, the cervix, uterus, vaginal walls, and insertion route (cervico-uterine canal) were contoured on MRI pre-BT. Next, 3D reconstruction of a virtual patient was created using the TPS. In cases with clinically non-visible cervical orifice, the entrance of insertion route was mapped on the surface of the reconstructed cervix in virtual speculum view. This was done by measuring the distances from virtual vaginal fornices to the entry point of the contoured cervical canal. Finally, the insertion route was divided into straight segments. Length and angle of each segment was recorded, and the total length of the insertion route calculated to select the optimal tandem length. Regions of perforation risk were identified and marked on the application route in representative MRI slices and virtual patient views. Insertion of the applicator under anesthesia was scheduled a week after pre-planning. Navigation parameters and visual reconstruction of the application route were followed during the procedure. Time to perform NAI (including pre-BT MRI) was
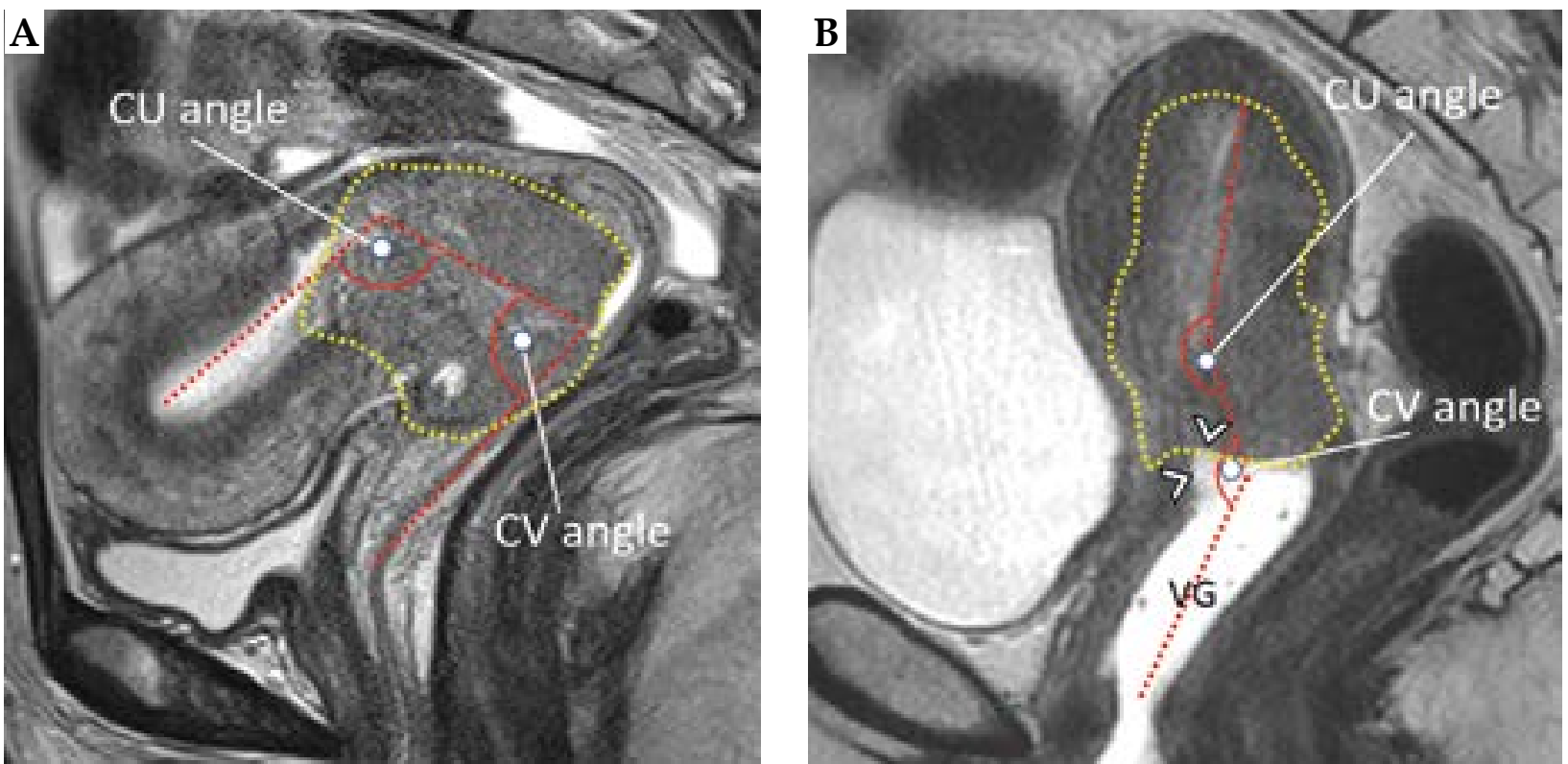

Fig. 2. Schematic representation of cervico-vaginal $(C V)$ and cervico-uterine $(C U)$ angles in two study cases on a sagittal T2w FSE magnetic resonance imaging (MRI). A) Initial MRI of a case with angles $<180^{\circ}$. B) Pre-brachytherapy MRI in a different case with cervico-uterine angle $>180^{\circ}$ (retroflected uterus). Open arrows - necrotic defect. VG - vaginal gel, used to unfold the vaginal walls and improve visualization. Distorted cervical canal was present in this example. Yellow dotted line - gross tumor volume 
estimated by the operator. Three-tiered scoring system was used to assess insertion difficulty: 1 - straightforward, non-complicated procedure; 2 - complicated procedure, requiring assistance of radiologist or gynecologist; 3 - procedure terminated due to difficulty or applicator false route.

\section{Statistical analysis}

Descriptive statistics were used to present frequencies of categorical tumor characteristics at diagnosis (histology and stage distribution) and pre-BT evaluation (risk factors for perforation). Continuous variables (time intervals, GTV sizes and angles) were presented by mean values with standard deviations. Statistical testing was limited to comparisons of a single variable: mean GTV on $\mathrm{MRI}_{\text {initial }}$ was compared with MRI $\mathrm{pre-BT}_{\text {and }}$ aRI $\mathrm{I}_{\text {pre-BT }}$ with $\mathrm{MRI}_{\mathrm{BT1}}$. Paired sample $t$-test was used and $p$-value of $<0.05$ was considered as the limit for statistical significance.

Table 1. Characteristics of tumors

\begin{tabular}{lcc} 
Tumor characteristic & $\begin{array}{c}\text { Number of } \\
\text { cases }(n)\end{array}$ & Proportion (\%) \\
\hline Histopathology & & \\
\hline Squamous carcinoma & 24 & 88.9 \\
\hline $\begin{array}{l}\text { Adeno- or adeno- } \\
\text { squamous carcinoma }\end{array}$ & 3 & 11.1 \\
\hline FIGO stage & 4 & 14.8 \\
\hline IB1-IB2 & 13 & 48.1 \\
\hline IIA-IIB & 6 & 22.2 \\
\hline IIIA-IIIB & 4 & 14.8 \\
\hline IVA-IVB & 18 & 66.7 \\
\hline Maximal tumor diameter & 9 & 33.3 \\
\hline$\geq 50 \mathrm{~mm}$ & &
\end{tabular}

FIGO - Federation Internationale de Gynécologie et d'Obstetrique

Table 2. Gross tumor volume (GTV), cervico-vaginal (CV) angle, and cervico-uterine (CU) angle. The differences between GTV sizes were statistically significant (initial magnetic resonance imaging [MRI] vs. pre-brachytherapy [BT] MRI: $p<0.01$ and pre-BT MRI vs. first BT fraction: $p=0.01$ )

\begin{tabular}{lccc} 
& \multicolumn{3}{c}{ MRI-based measurement (mean [SD]) } \\
\cline { 2 - 4 } & Initial & Pre-BT & $1^{\text {st }}$ BT fraction \\
\hline GTV $\left(\mathrm{cm}^{3}\right)$ & $70.2(47.9)$ & $17.8(18.9)$ & $10.3(9.1)$ \\
\hline CV angle $\left(^{\circ}\right)$ & $131(32)$ & $132(33)$ & NA \\
\hline CU angle $\left(^{\circ}\right)$ & $169(44)$ & $175(54)$ & NA
\end{tabular}

$N A$ - not applicable (at BT, angles correspond to applicator geometry)

\section{Results}

\section{Patients and tumors}

Thirty patients were considered for inclusion in the study. Two were excluded due to incomplete imaging data and one additional case due to non-standard treatment protocol (weekly BT during EBRT). Twenty-seven patients who underwent a total of 98 applicator insertions were eligible for analysis. Median patient age was 43 (range, 32-67) years. Characteristics of tumors at time of diagnosis are summarized in Table 1 . The average interval from MRI simulator to EBRT and from pre-BT MRI to BT was $9( \pm 7)$ and $7( \pm 5)$ days, respectively. The interval from MRI simulator to pre-BT MRI was $42( \pm 9)$ days. Mean GTV sizes, cervico-vaginal angles, and cervico-uterine angles are presented in Table 2. GTV reduced significantly during EBRT from $70.2 \pm 47.9 \mathrm{~cm}^{3}$ on $\mathrm{MRI}_{\text {initial }}$ to $17.8 \pm 18.9$ on MRI $\mathrm{Mre}_{\text {-BT }}(p<0.01)$, and further to $10.3 \pm 9.1 \mathrm{~cm}^{3}$ on $\mathrm{MRI}_{\mathrm{BT1}}(p=0.01)$.

\section{Risk factors for perforation}

In $11(41 \%)$ patients, there were no risk factors for perforation on pre-BT assessment. Remaining 16 (59\%) cases had at least one risk factor, most commonly distorted cervical canal in 12 (44\%), followed by necrosis in $9(33 \%)$, and uterus in RVF in 8 (30\%) patients (Figure 3). Inability to identify cervical orifice on pre-BT evaluation and age over 60 years were less common (Figure 3). Among the 8 patients with uterus in RVF position, cervico-uterine and cervico-vaginal angle was $\geq 180^{\circ}$ in 8 and 2 cases, respectively (Figure 4). In 6 out of 8 patients with RVF uterus, this finding was depicted already on MRI $\mathrm{initial}_{\text {. In }}$ remaining 2, cervico-vaginal and/or cervico-uterine angle was less than $180^{\circ}$ on $\mathrm{MRI}_{\text {initial, }}$, but shifted to $\geq 180^{\circ}$ on $\mathrm{MRI}_{\text {pre-BT }}$ (Figure 4). Analysis of daily cone beam CT in these 2 cases revealed that the shift occurred on $18^{\text {th }}$ and $5^{\text {th }}$ fraction of EBRT, respectively. In both cases, the uterus remained in RVF position until the end of treatment. Mean cervico-vaginal and cervico-uterine angles at $\mathrm{MRI}_{\text {initial }}$ and MRI $\mathrm{pre-BT}$ are shown in Table 2.

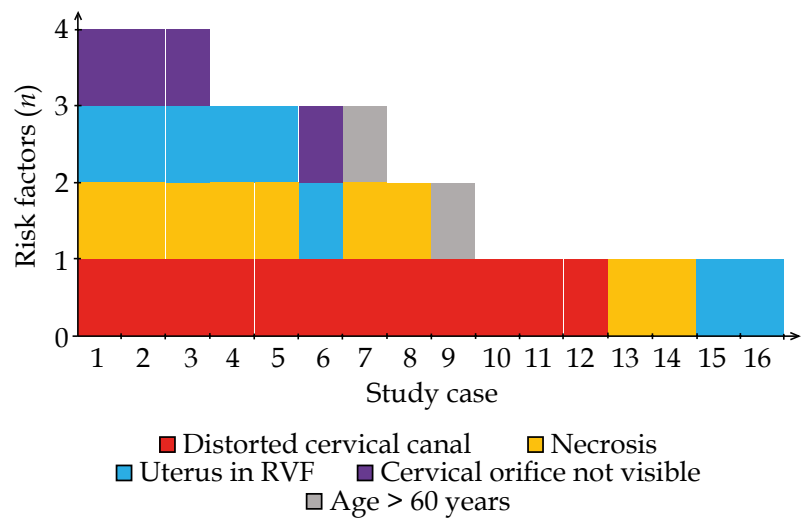

Fig. 3. Distribution of findings in 16 cases with risk factors for perforation on pre-brachytherapy evaluation. RVF retroversion and/or retroflection 
A

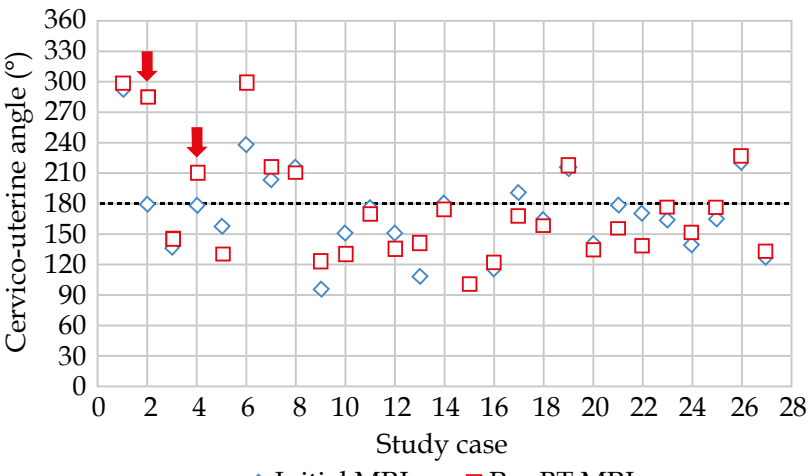

$\diamond$ Initial MRI $\quad \square$ Pre-BT MRI
B

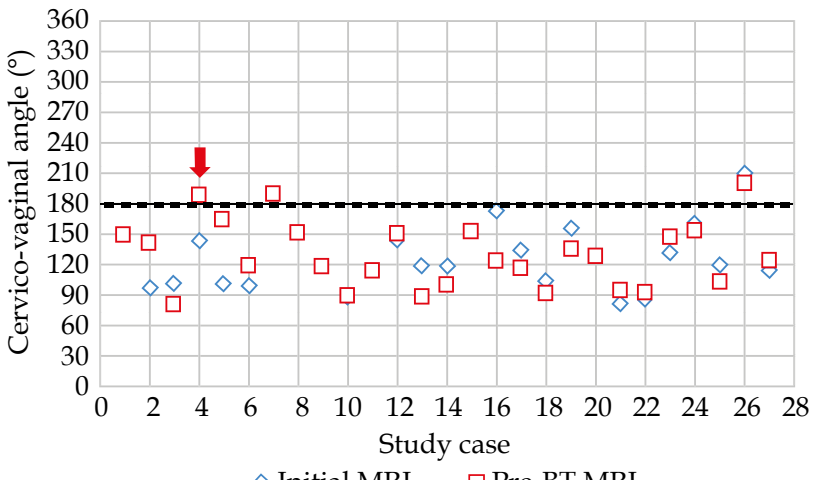

$\diamond$ Initial MRI $\quad \square$ Pre-BT MRI

Fig. 4. Cervico-uterine and cervico-vaginal angle in individual study cases, measured from initial and pre-brachytherapy magnetic resonance imaging (MRI). Red arrows mark the cases in which the angle changed from $<180^{\circ}$ on initial MRI to $\geq 180^{\circ}$ on pre-brachytherapy MRI

\section{Feasibility of magnetic resonance imaging-guided navigation for applicator insertion}

Nine $(33 \%)$ patients who presented with $\geq 2$ risk factors underwent MRI guided NAI. Systematic use of the navigation parameters increased the operator confidence during applicator insertion. Operator-reported difficulty of NAI-based BT was favorable: applicator insertion was straightforward and non-complicated in all cases (difficulty score 1). Assistance of gynecologist or radiologist was not required in any case. There were no perforations, subserosal insertions or other acute complications of BT procedure. Figure 5 shows the examples of NAI pre-planning in 2 cases. Average additional time required to perform NAI was estimated at 105 minutes. Since off-line MRI guided NAI was performed in parallel with treatment during the $5^{\text {th }}$ week of EBRT, it did not interfere with our routine fractionation schedule and had no impact on the overall treatment time.

\section{Discussion}

We reported on the frequency of risk factors for uterine perforation in our patient cohort, based on combined pre-insertion clinical and MRI evaluation. One third of our patients presented with two or more risk factors and consequently underwent systematic MRI guided pre-planning of IC applicator placement. MRI guided NAI was fast and feasible and led to uncomplicated insertion in all cases.

We identified no cases of uterine perforation in our study. Perforation rates prior to implementation of MRI assisted IGABT were not analyzed. It needs to be emphasized that the present study and sample size were not designed to detect the impact of MRI guided navigation on the incidence of these relatively rare, but potentially serious events. The incidence of perforations reported in the literature ranges from approximately $2-14 \%$ of insertions in different series $[8,9,10,11]$. In a recent large mono-institutional report, 428 IGABT procedures in 219 patients were analyzed retrospectively [8]. No pre-planning of IC insertion was performed, but ultrasound guidance was used in challenging cases. Uterine perforation was identified in $10(4.6 \%)$ patients and $13(3 \%)$ procedures [8]. Bahadur et al. recently reported on the incidence of applicator misplacement in a cohort of 82 patients who underwent 231 insertions. Perforation was identified in $12(14.6 \%)$ patients and $14(6 \%)$ applications. Subserosal insertion of the tandem was found in further $12(14.6 \%)$ patients and $20(8.6 \%)$ applications. The authors concluded that the lack of ultrasound guidance in their study was the most likely reason for higher incidence of applicator misplacement [10]. In another report, 124 sequential insertions were performed in 114 patients without pre-insertion imaging or on-line ultrasound guidance. The incidence of perforation, detected on post-insertion CT was $13.7 \%$ [9]. Several studies reported on the role of intraoperative ultrasound during BT applications, and this approach is currently considered gold standard for on-line guidance of IC applicator insertion [8,11,12,13,14,15,16]. For difficult or questionable insertions, even direct endoscopic evaluation at the time of tandem insertion was proposed for verification of tandem position [23], but this is not widely used. The need for ultrasound-based real time guidance is especially important in challenging cases with risk factors for perforation. In the study by Segedin et al., the use of intraoperative transabdominal and/ or transrectal ultrasound in high-risk patients who had prior perforation, enabled optimal applicator placement on consequent insertions in all cases [8]. Mayr et al. reported on 33 ultrasound-guided applicator insertions in 18 patients with retroverted uterus. Anteversion of the inserted tandem and uterus was achieved in all procedures, and there were no cases of perforation [12]. In our present study, there were 8 patients ( 30 procedures) with the

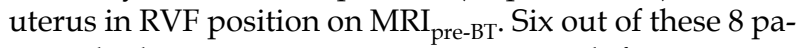
tients had two or more accompanying risk factors, most commonly distorted cervical canal and necrosis. Using the information from MRI $\mathrm{pre-BT}_{\text {-BT }}$ and systematic off-line MRI guided NAI, anteversion of the uterus and optimal applicator placement were achieved in all cases without ultrasound assistance. These results indicate that comprehensive consideration of all imaging and clinical data before the applicator insertion can importantly complement 

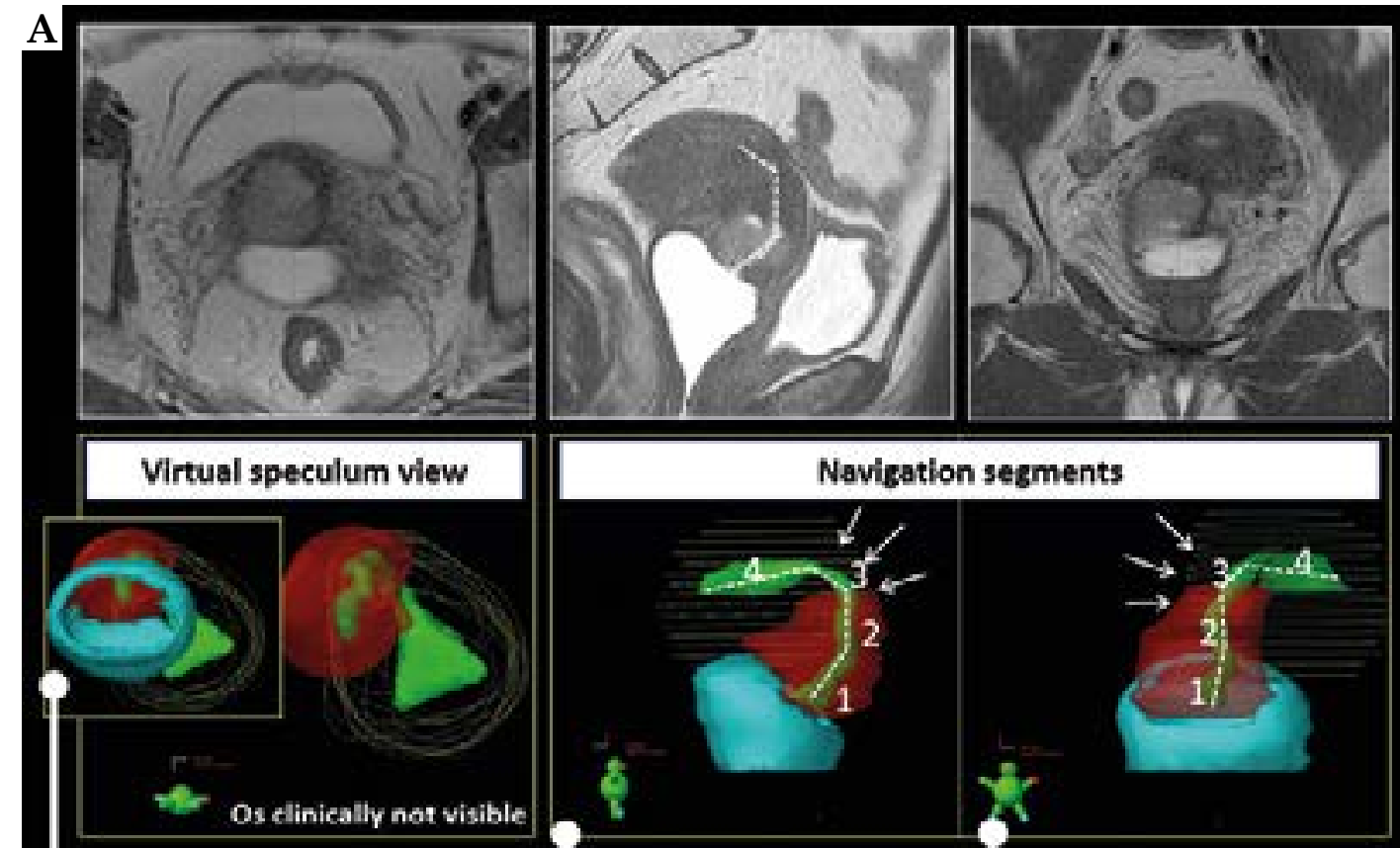

Cervical os coordinates:

$8 \mathrm{~mm}$ from ant. fornix

$20 \mathrm{~mm}$ from post. fornix

$12 \mathrm{~mm}$ from left fornix

$20 \mathrm{~mm}$ from right fornix
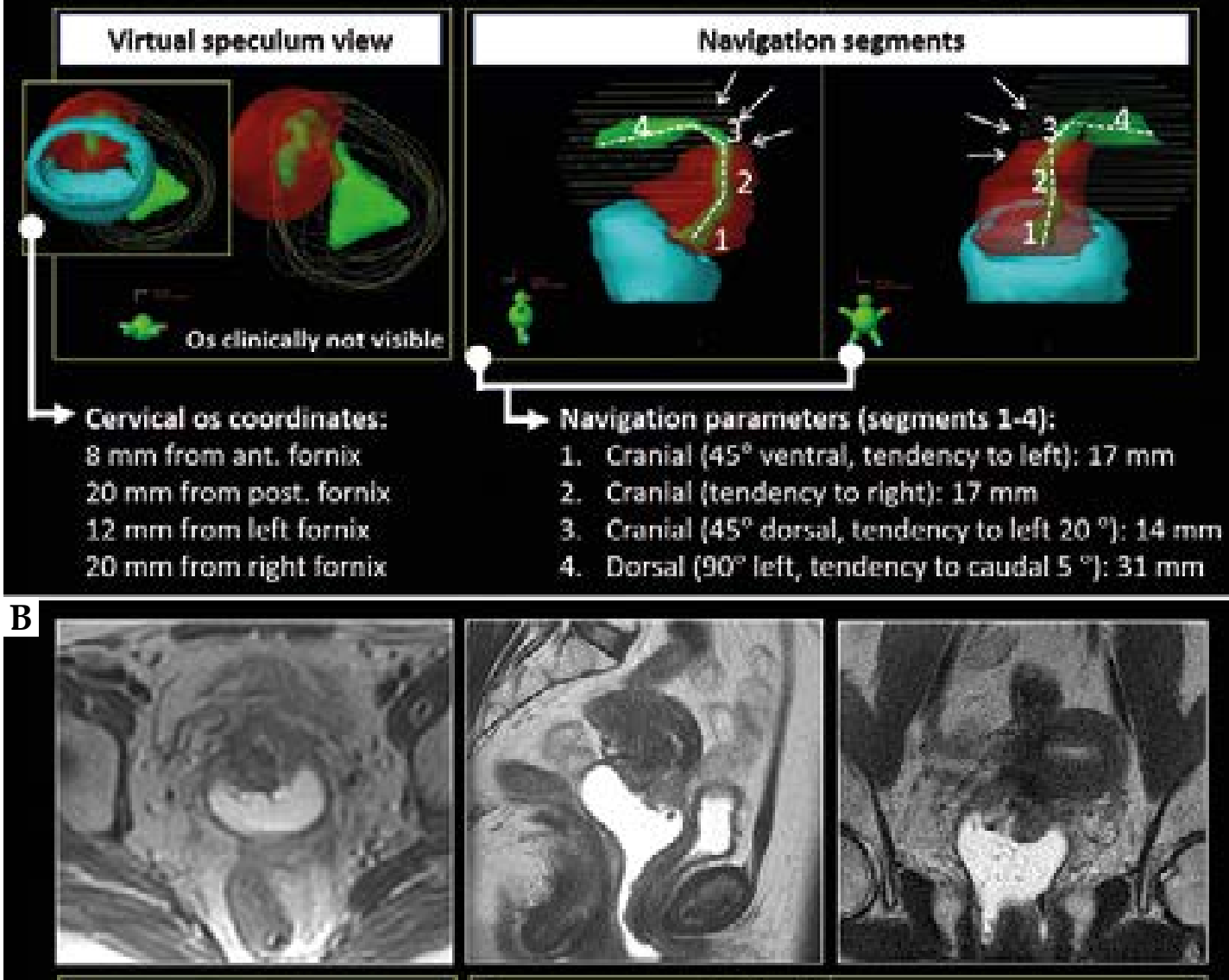

Virtual speculum view
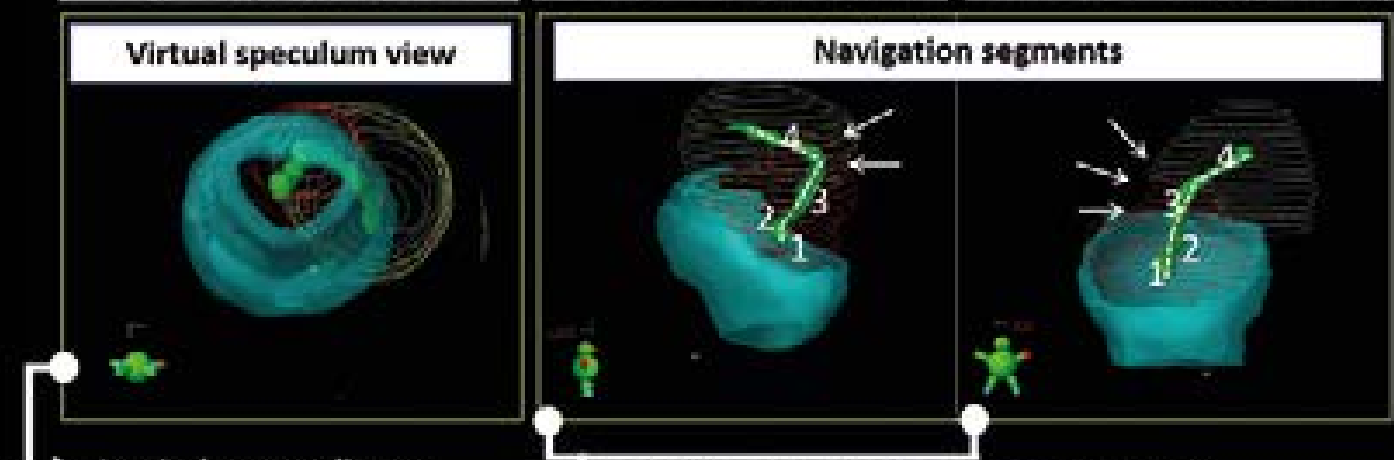

Cervical os coordinates:

Visible clinically.

Navigation not needed.

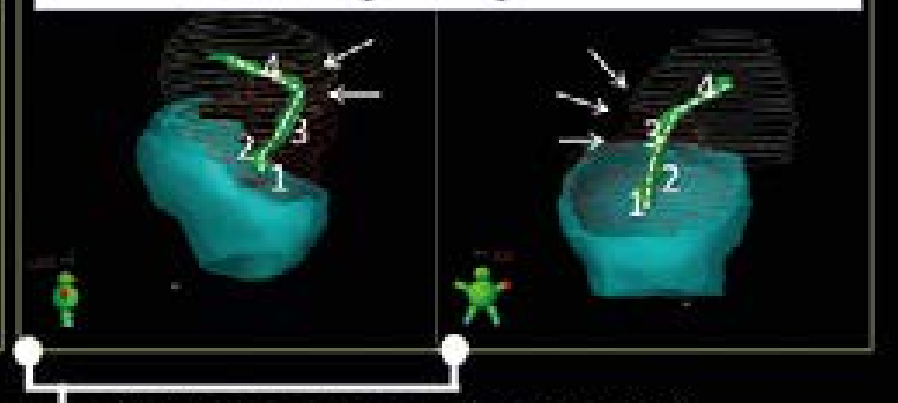

Navigation parameters (segments 1-4):

1. Cranial (straight, tendency left): $5 \mathrm{~mm}$

2. Cranial ( $30^{\circ}$ anterior, tendency to left): $5 \mathrm{~mm}$

3. Cranial $\left(35^{\circ}\right.$ anterior, $30^{\circ}$ left:): $25 \mathrm{~mm}$

4. Cranial $\left(45^{\circ}\right.$ posterior, $45^{\circ}$ left) : $25 \mathrm{~mm}$

Fig. 5. Magnetic resonance imaging-guided navigation of applicator insertion in two study examples. Yellow - uterus, red - cervix, green - cervico-uterine canal, cyan - vaginal walls, white dotted lines - navigation segments, white arrows - perforation risk 
other methods of image guidance, including intraoperative ultrasound. When applied in the systematic context of MRI guided NAI, the pre-planning of IC insertion may even have a potential as an alternative method to on-line guidance. However, this is relevant only for institutions, which have implemented MRI based IGABT, but have limited access to intraoperative ultrasound.

We used information from MRI $\mathrm{pre}_{\mathrm{BT}}$ to guide the IC \pm IS insertion in all cases. Nevertheless in cases with two or more risk factors for perforation, the formal systematic procedure of MRI guided NAI was followed (Figures 1 and 5). Several studies demonstrated that careful pre-planning of applicator insertion leads to optimal results of gynecological IGABT [24,25,26,27]. Different approaches to pre-planning were described in the literature. Their complexity varies from assessment of pre-treatment MRI alone [24] to geometric and dosimetric pre-planning, based on of pre-BT MRI with the applicator in place $[25,26,27]$. These methods refer exclusively to individualization of interstitial component of BT applications. The innovative method of off-line MRI guided NAI proposed in our present report enables dedicated off-line pre-planning of IC component. In our experience, off-line MRI guided NAI was fast and feasible, and increased operator confidence during dilatation of cervical canal and applicator insertion. It resulted in optimal placement of IC applicator even in cases with several risk factors for uterine perforation. Radiation oncologist with extensive experience in MRI-guided BT reviewed the MR images in our study, identifying no perforations or subserosal insertions. In view of unequivocal findings in all cases, second opinion from a radiologist was not sought. However, access to consultation with a diagnostic radiologist is strongly advised in clinical practice to minimize uncertainties, especially at an early phase of the IGABT learning curve. The importance of adequate radiology training of oncologists, practicing MRI assisted IGABT, cannot be over-emphasized [22].

Several authors attempted to identify risk factors from post-insertion sectional images with the applicator in place. In the study by Segedin et al., age over 60 years, distorted cervical canal, tumor necrosis, and retroflected uterus were present in $8,6,4$, and 3 out of 10 patients with perforation, respectively [8]. Combination of two or more of risk factors or age above 60 years was present in $90 \%$ of cases with perforation [8]. Barnes et al. identified post-insertion physician concern $(p<0.001)$, age greater than or equal to 60 years $(p=0.0019)$, and tumor size $(p=0.016)$ as significant predictors for uterine perforation [9]. In the study by Bahadur et al., stage was the only significant predictor of suboptimal insertion $(p=0.005)$ [10]. Of note, image based assessment of risk factors in these studies was performed only after the applicator insertion. But to reduce the chance of perforation and its negative consequences, pre-insertion identification of patients at risk is required. To our knowledge, our present study is the first to report on a systematic use of MRI ${ }_{\text {pre-BT }}$ for pre-operative risk assessment. Some predictors, such as age and tumor stage are readily determined from pre-treatment diagnostic imaging and clinical findings at diagnosis and before BT. However, tumor size and topography, amount of necrosis and fibrosis, topography of uterus and cervix, changes during EBRT, and the response to treatment differ from patient to patient $[24,28,29,30,31]$. Therefore, some of the factors that were not present at diagnosis, but became apparent before BT, can be identified only by means of additional pre-insertion imaging. Due its soft-tissue depiction capability, MRI is best suited modality for this purpose.

The risk factors suggested by Segedin et al. [8] were used in our study. We found at least one risk factor for uterine perforation in $16(59 \%)$ cases, and two or more factors in $9(33 \%)$ cases on pre-BT evaluation. Necrosis, distorted cervical canal, and retroversion/retroflection of the uterus were encountered most frequently (Figure 3 ). The CU and/or $\mathrm{CV}$ angle $\geq 180^{\circ}$ alone or in combination with other risk factors was identified in $8(30 \%)$ of cases. In two of these patients, the uterus was anteflected/ anteverted at the start of treatment, but shifted to retroflection/retroversion during EBRT and remained in this position until the end of BT. These findings underscore the importance of MRI pre-BT for timely identification of unexpected findings at BT. According to our findings, MRI guided NAI could be considered in about one third of patients, if presence of two risk factors was used as the threshold. Setting the threshold at a single risk factor would imply the use of NAI in up to approximately $60 \%$ of clinical scenarios. However, the frequencies suggested here must be interpreted with caution because of the small study sample used in our study. Nevertheless, it should be noted that we included all eligible cases treated at our Centre, since implementation of MRI based IGABT in 2013. Furthermore, tumor stages and dimensions at diagnosis and BT, and the pattern of volumetric GTV regression during EBRT (Table 1 and 2) were comparable to reports of others [28]. In summary, our results may be regarded as a proof of concept for MRI-based preoperative assessment of perforation risk and consequent MRI guided NAI in challenging cases. Further analysis of clinical utility and cost-effectiveness of this promising technique, based on a larger number of patients, is warranted.

\section{Conclusions}

Pre-brachytherapy MRI enables identification of patients at risk for uterine perforation during cervix cancer BT. Two or more risk factors were found in one third of our patients. Systematic pre-planning of intracavitary insertion with off-line MRI guided navigation is fast and feasible, increases operator confidence during challenging applications, and may reduce the risk of perforation. It can complement the standard approach of on-line guidance with intraoperative ultrasound. Our results may be regarded as a proof of concept regarding feasibility of MRI guided NAI. Further studies are warranted to confirm the potential positive clinical impact of MRI guided navigation on safety of BT applications.

\section{Disclosure}

Authors report no conflict of interest. 


\section{References}

1. Tanderup K, Lindegaard JC, Kirisits C et al. Image Guided Adaptive Brachytherapy in cervix cancer: A new paradigm changing clinical practice and outcome. Radiother Oncol 2016; 120: 365-369.

2. Sturdza A, Pötter R, Fokdal LU et al. Image guided brachytherapy in locally advanced cervical cancer: Improved pelvic control and survival in RetroEMBRACE, a multicenter cohort study. Radiother Oncol 2016; 120: 428-433.

3. Fokdal L, Sturdza A, Mazeron R et al. Image guided adaptive brachytherapy with combined intracavitary and interstitial technique improves the therapeutic ratio in locally advanced cervical cancer: Analysis from the retroEMBRACE study. Radiother Oncol 2016; 120: 434-440.

4. Harkenrider MM, Shea SM, Wood AM et al. How one institution overcame the challenges to start an MRI-based brachytherapy program for cervical cancer. J Contemp Brachytherapy 2017; 9: 177-186.

5. Otter S, Franklin A, Ajaz M, Stewart A. Improving the efficiency of image guided brachytherapy in cervical cancer. J Contemp Brachytherapy 2016; 8: 557-565.

6. Carvalho Hde A, Mendez LC, Stuart SR et al. Implementation of image-guided brachytherapy (IGBT) for patients with uterine cervix cancer: a tumor volume kinetics approach. J Contemp Brachytherapy 2016; 8: 301-307.

7. Yip WWL, Wong JSY, Lee VWY et al. Throwing the dart blind-folded: comparison of computed tomography versus magnetic resonance imaging-guided brachytherapy for cervical cancer with regard to dose received by the 'actual' targets and organs at risk. J Contemp Brachytherapy 2017; 9: 446-452.

8. Segedin B, Gugic J, Petric P. Uterine perforation - 5-year experience in 3-D image guided gynaecological brachytherapy at Institute of Oncology Ljubljana. Radiol Oncol 2013; 47: 154160.

9. Barnes EA, Thomas G, Ackerman I et al. Prospective comparison of clinical and computed tomography assessment in detecting uterine perforation with intracavitary brachytherapy for carcinoma of the cervix. Int J Gynecol Cancer 2007; 17: 821-826.

10. Bahadur YA, Eltaher MM, Hassouna AH et al. Uterine perforation and its dosimetric implications in cervical cancer high-dose-rate brachytherapy. J Contemp Brachytherapy 2015; 7: 41-47.

11. Granai CO, Doherty F, Allee P et al. Ultrasound for diagnosing and preventing malplacement of intrauterine tandems. Obstet Gynecol 1990; 75: 110-113.

12. Mayr NA, Montebello JF, Sorosky JI et al. Brachytherapy management of the retroverted uterus using ultrasound-guided implant applicator placement. Brachytherapy 2005; 4: 24-29.

13. Watkins JM, Kearney PL, Opfermann KJ et al. Ultrasound-guided tandem placement for low-dose-rate brachytherapy in advanced cervical cancer minimizes risk of intraoperative uterine perforation. Ultrasound Obstet Gynecol 2011; 37: 241-244.

14. Sharma DN, Rath GK, Thulkar S et al. Use of transrectal ultrasound for high dose rate interstitial brachytherapy for patients of carcinoma of uterine cervix. J Gynecol Oncol 2010; 21: $12-17$.

15. Small W Jr, Strauss JB, Hwang CS et al. Should uterine tandem applicators ever be placed without ultrasound guidance? No: a brief report and review of the literature. Int J Gynecol Cancer 2011; 21: 941-944.

16. Davidson MT, Yuen J, D'Souza DP et al. Optimization of high-dose-rate cervix brachytherapy applicator placement: The benefits of intraoperative ultrasound guidance. Brachytherapy 2008; 7: 248-253.
17. Dogan N, King S, Emami B et al. Assessment of different IMRT boost delivery methods on target coverage and normal tissue sparing. Int J Radiat Oncol Biol Phys 2003; 57: 1480-1491.

18. Feng CH, Hasan Y, Kopec M, Al-Hallaq HA. Simultaneously integrated boost (SIB) spares OAR and reduces treatment time in locally advanced cervical cancer. J Appl Clin Med Phys 2016; 19: 76-89.

19. Haie-Meder C, Pötter R, van Limbergen E et al. Recommendations from Gynaecological (GYN) GEC-ESTRO Working Group (I): concepts and terms in 3D image based 3D treatment planning in cervix cancer brachytherapy with emphasis on MRI assessment of GTV and CTV. Radiother Oncol 2005; 74: 235-245.

20. Dimopoulos JC, Petrow P, Tanderup K et al. Recommendations from Gynaecological (GYN) GEC-ESTRO Working Group (IV): Basic principles and parameters for MR imaging within the frame of image based adaptive cervix cancer brachytherapy. Radiother Oncol 2012; 103: 113-122.

21. Torfeh T, Hammoud R, Perkins G et al. Characterization of $3 \mathrm{D}$ geometric distortion of magnetic resonance imaging scanners commissioned for radiation therapy planning. Magn Reson Imaging 2016; 34: 645-653.

22. Petric P, Al-Hammadi N. MRI findings at image guided adaptive cervix cancer brachytherapy: radiation oncologist's perspective. J Contemp Brachytherapy 2014; 6: 215-222.

23. Irvin $\mathrm{W}$, Rice $\mathrm{L}$, Taylor $\mathrm{P}$ et al. Uterine perforation at the time of brachytherapy for carcinoma of the cervix. Gynecol Oncol 2003; 90: 113-122.

24. Schmid MP, Fidarova E, Pötter R et al. Magnetic resonance imaging for assessment of parametrial tumour spread and regression patterns in adaptive cervix cancer radiotherapy. Acta Oncol 2013; 52: 1384-1390.

25. Fokdal L, Tanderup K, Hokland SB et al. Clinical feasibility of combined intracavitary/interstitial brachytherapy in locally advanced cervical cancer employing MRI with a tandem/ring applicator in situ and virtual preplanning of the interstitial component. Radiother Oncol 2013; 107: 63-68.

26. Petric P, Hudej R, Hanuna O et al. MRI-assisted cervix cancer brachytherapy pre-planning, based on application in paracervical anaesthesia: final report. Radiol Oncol 2014; 48: 293-300.

27. Nomden CN, De Leeuw AAC, Moerland MA et al. Clinical use of the Utrecht applicator for combined intracavitary/ interstitial brachytherapy treatment in locally advanced cervical cancer. Int J Radiat Oncol Biol Phys 2012; 82:1424-1430.

28. Dimopoulos JC, Schirl G, Baldinger A et al. MRI assessment of cervical cancer for adaptive radiotherapy. Strahlenther Onkol 2009; 185: 282-287.

29. Mayr NA, Wang JZ, Lo SS et al. Translating response during therapy into ultimate treatment outcome: a personalized 4-dimensional MRI tumor volumetric regression approach in cervical cancer. Int J Radiat Oncol Biol Phys 2010; 76: 719-727.

30. Wang JZ, Mayr NA, Zhang D et al. Sequential magnetic resonance imaging of cervical cancer: the predictive value of absolute tumor volume and regression ratio measured before, during, and after radiation therapy. Cancer 2010; 116: 5093-5101.

31. Mongula J, Slangen B, Lambregts D et al. Predictive criteria for MRI-based evaluation of response both during and after radiotherapy for cervical cancer. J Contemp Brachytherapy 2016; 8: 181-188. 